\title{
Outcomes from the first assisted reproduction program for HIV- serodiscordant couples in Australia
}

Michelle L Giles MB BS, FRACP, PhD, Infectious Diseases Physician ${ }^{1,2}$

Shlomi Barak FRANZCOG Fertility Specialist Reproductive Services ${ }^{1,3}$

Gordon Bake

MD, PhD, FRACP, Professor (Reproductive Biology) $)^{1,4}$

Sepehr Tabriz BS, MS, PhD, Senior Research Scientist, Department of Microbiology and Infectious Diseases

Vicki Greengrass BSc(Hons) Supervising Scientist 1,

Harold Bourne BSc(Hons), MRepSc, Supervising Scientist Reproductive Services ${ }^{1,3}$

Gary N Clarke BS, MS, DSC,

Scientist in Charge, Department of Andrology

Suellen A Peak BA, BSW Fertility Counsellor

Jennifer F Hoy MB BS, FRACP PhD, Head, Victorian HIV Service 2,6

Penelope Foste MRCOG, FRANZCOG Fertility Specialist Reproductive Services

Rachael L Knight MB BS, BMedSci, FRANZCOG

Gynaecologist and Fertility Specialist Reproductive Services ${ }^{1,3}$

on behalf of the CVI Study Group

1 Royal Women's Hospital Melbourne, VIC

2 The Alfred Hospital Melbourne, VIC

3 Melbourne IVF Melbourne, VIC

4 University of Melbourne, Melbourne, VIC

5 Centre for Virology, Burnet Institute, Melbourne, VIC.

6 Monash University. Melbourne, VIC

m.giles@alfred.org.au

MJA 2011; 195: 599-601 doi: 10.5694/mjall.10156

Editorial p 564 Research p 594 bout 33 million people worldwide are living with HIV, the majority of whom are of reproductive age. ${ }^{1}$ The introduction of combination antiretroviral therapy (ART) has led to a significant reduction in morbidity and mortality for HIV-positive individuals. This has dramatically changed the approach many countries take towards reproductive choices for HIV-positive people.

HIV-positive individuals may wish to have children. ${ }^{2-6}$ Options to achieve pregnancy for HIV-serodiscordant couples (where one partner has HIV infection and the other does not) include unprotected sexual intercourse, self-insemination, intrauterine insemination and in-vitro fertilisation (IVF)/intracytoplasmic sperm injection (ICSI). The principal considerations when evaluating these methods for couples affected by HIV include risk of horizontal transmission of HIV to the uninfected partner, efficacy of achieving pregnancy, access to treatment, coexisting subfertility, and personal choice.

In 2008, the Swiss Federal Commission for HIV/AIDS stated that, in the absence of other sexually transmitted infections, HIV-positive people who were using effective ART were not infectious. ${ }^{7}$ In response to this controversial statement, some national public health bodies reasserted the need for consistent condom use, $^{8}$ as did researchers from Australia who applied mathematical modelling to conclude that the risk of transmission in heterosexual partnerships in the presence of effective treatment is low but not zero. ${ }^{9}$

Here, we report the outcomes for HIV-serodiscordant couples attending an assisted reproduction program between 2003 and June 2010 in Australia.

\section{Methods}

Assisted reproductive techniques have been offered to HIV-positive men with

Abstract

Objective: To describe the clinical outcomes for all HIV-serodiscordant couples attending an assisted reproduction program.

Design, setting and participants: Retrospective review of demographic, clinical and outcome data for all HIV-serodiscordant couples who attended an assisted reproduction program at a tertiary hospital in Melbourne, between its commencement in 2003 and June 2010.

Main outcome measures: Pregnancies, miscarriages, births, HIV transmission to the HIV-negative partner, semen quality and detection of HIV (HIV RNA and HIV DNA) in semen.

Results: As of June 2010, 39 HIV-positive clients had proceeded to assisted reproduction after the initial consultation in the program. There were 162 completed cycles, with 26 pregnancies (clinical pregnancy rate per cycle, 16.2\% for HIV-positive men with an HIV-negative partner, and 15.4\% for HIV-positive women). Of all 222 tested semen samples, 18 (8\%) had HIV RNA detected despite these men receiving antiretroviral therapy and having an undetectable HIV viral load in plasma. Sperm velocity was significantly lower in HIV-positive clients receiving combination antiretroviral therapy than in a control group of recipient-recruited sperm donors $(P=0.01)$; there were no other significant differences in sperm quality between the two groups. No HIV transmission to babies or HIV-negative partners occurred.

Conclusion: Our findings show detectable HIV in $8 \%$ of semen samples from men with an undetectable HIV viral load in plasma, but confirm the safety of assisted reproduction for HIV-serodiscordant couples within a program with strict protocols for HIV treatment and testing of all semen before use.

HIV-negative female partners at the Royal Women's Hospital in Melbourne since 2003, and to HIV-positive women since 2006. Details of the program have previously been published, ${ }^{10}$ as have details of the laboratory process and semen HIV testing. ${ }^{11}$

To participate in the program, HIVpositive men must have a plasma viral load below the detection limits of the assay $(<50$ copies $/ \mathrm{mL})$ for a minimum of 2 months before providing two semen samples that undergo HIV RNA quantification.

Once the HIV-positive man's two semen samples are cleared (ie, with undetectable HIV RNA), he provides further semen samples that are cryopreserved, tested for HIV RNA and HIV DNA and used for future clinical treatment. The HIV proviral DNA assay is performed on the pellet that results from centrifugation of $400 \mu \mathrm{L}$ of semen diluted with Tyrode's solution (JRH Biosciences, Lenexa, Kan, USA).

Semen analysis ${ }^{12}$ is performed for all male clients attending the program and measures volume, sperm count, motility, velocity and morphology.
The choice of treatment offered (intrauterine insemination or IVF/ ICSI) depends on the woman's age, reproductive history, results of fertility investigation, and the quality of the prepared sperm. After treatment, HIV testing is performed at 14 days, 6 weeks, 12 weeks, and 6 months if not pregnant or 32 weeks' gestation and at birth of the baby if pregnant.

Ethics approval was not required for this study as it was a retrospective audit.

\section{Statistical analysis}

We made statistical comparisons between the HIV-positive men in our study group and a control group of recipient-recruited sperm donors. The continuous variables of age and semen characteristics were compared using the non-parametric KolmogorovSmirnov two-sample test. Statistical analysis was performed using Statgraphics, version 5 (Statistical Graphics Corp, Warrenton, Va, USA).

\section{Results}

As of June 2010, 39 HIV-positive clients had proceeded to assisted repro- 
1 Semen analysis for HIV-positive men and control group of recipient-recruited sperm donors

\begin{tabular}{lccc} 
& \multicolumn{2}{c}{ Median (range) } & \multirow{2}{*}{ Variable } \\
\cline { 2 - 3 } & Control group $(n=114)$ & HIV group $(n=29 *)$ & $P$ \\
\hline Man's age (years) & $38.8(23.4-64.8)$ & $36.7(26.6-51.9)$ & 0.37 \\
Abstinence (days) & $3.5(1-10)$ & $4.0(1-28)$ & 0.06 \\
Delay to testing $($ hours) & $0.7(0.3-1.7)$ & $0.5(0.3-1.1)$ & $<0.001$ \\
Semen volume $(\mathrm{mL})$ & $3.2(0.3-9.9)$ & $3.4(1.4-5.2)$ & 0.44 \\
Sperm count $\left(10^{6} / \mathrm{mL}\right)$ & $84(13-750)$ & $62(17-313)$ & 0.20 \\
Sperm motility $(\%)$ & $49(20-84)$ & $50(14-77)$ & 0.86 \\
Sperm velocity $(\mu / \mathrm{sec})$ & $42(22-66)$ & $35(17-59)$ & 0.01 \\
Morphology $(\%$ abnormal) & $83(46-98)$ & $78(61-95)$ & 0.43 \\
\hline * Morphology data missing for one man, sperm velocity missing for two men. &
\end{tabular}

\section{Clients and outcomes of assisted reproduction program, 2003 - June 2010}

HIV-positive man with HIV-positive woman with HIV-negative female partner HIV-negative male partner

\begin{tabular}{|lcc|}
\hline Number of patients* & 27 & 8 \\
\hline Completed cycles & 136 & 26 \\
\hline Pregnancies with an outcome & 22 & 4 \\
\hline Miscarriages & 7 & 0 \\
\hline Babies born & 15 & 4 \\
\hline Ongoing pregnancies & 2 & 1 \\
\hline Clinical pregnancy ${ }^{\dagger}$ rate per cycle & $16.2 \%$ & $50 \%$ \\
\hline $\begin{array}{l}\text { Clinical pregnancy }{ }^{\dagger} \text { rate per } \\
\text { number of patients }\end{array}$ & $81.5 \%$ & \\
\hline
\end{tabular}

* Those with complete data who proceeded with treatment. $\dagger$ Fetal heartbeat at 6 weeks after embryo transfer or intrauterine insemination.

duction after the initial consultation in the program: $29 \mathrm{HIV}$-positive men with an HIV-negative female partner, and 10 HIV-positive women (eight with an HIV-negative male partner and two using donor sperm).

For the 37 clients (27 HIV-positive men, 10 HIV-positive women) with complete data available, the median year of HIV diagnosis was 1999 (range, 1984-2006). Of these 37 clients, four had a history of an AIDSdefining illness, 32 had a plasma HIV viral load $<400$ copies/mL at their first consultation, and five were ARTnaive. Four clients were commenced on ART solely to meet the program's protocol requirement of undetectable plasma HIV viral load for 2 months before providing semen samples for testing, and not because of standard immunological parameters. One HIVpositive man had persistently undetectable plasma HIV viral load, so was not commenced on ART. Nine clients were co-infected with hepatitis $\mathrm{C}$ and four with hepatitis B virus.

Of the $27 \mathrm{HIV}$-positive clients with complete semen analysis results and
ART data, 18 had at least one abnormality reported, and seven of these had more than one abnormality. Comparison of semen results from all 29 HIV-positive men with the control group of 114 recipient-recruited sperm donors indicated that the HIVpositive men had statistically significantly lower straight-line sperm velocity $(P=0.01)$ (Box 1$)$. There were no significant differences in sperm count, motility, morphology or ejaculate volume. The median ages and periods of sexual abstinence before provision of semen samples of the HIV-positive and control groups were not significantly different.

Of a total of 222 semen samples tested for HIV RNA (including the initial two samples provided by each man), 18 (8\%) had HIV RNA quantified at between 100 and 1400 copies/ $\mathrm{mL}$, despite patients maintaining concomitant plasma viral loads of $<50$ copies/mL with combination ART.

There were 162 completed treatment cycles: 86 intrauterine insemination (53\%) and $76 \mathrm{IVF}$, of which 30 (19\%) were ICSI, 28 (17\%) were fro- zen embryo transfers and 18 (11\%) were non-ICSI. These cycles resulted in 26 pregnancies, equating to a clinical pregnancy rate per cycle of $16.2 \%$ for HIV-negative partners of HIVpositive men and $15.4 \%$ for HIV-positive women (Box 2). There were seven miscarriages and 19 babies born. There were no cases of HIV transmission to the baby or HIV-negative partner.

\section{Discussion}

Although we found detectable HIV viral load in $8 \%$ of semen samples from men who were receiving combination ART and had an undetectable HIV viral load in plasma, our study confirms the safety of assisted reproduction for HIV-serodiscordant couples within a program with strict protocols for HIV treatment and testing of all semen before use.

The clinical pregnancy rate per cycle in our study was $16.2 \%$ for HIVpositive men with an HIV-negative partner and $15.4 \%$ for HIV-positive women. In comparison, the clinical pregnancy rate from 61929 assisted reproductive technology treatment cycles undertaken for HIV-negative couples in Australia and New Zealand in 2008 was $22.6 \%$. $^{13}$

For HIV-positive men, there are few options for biological parenthood that carry no risk of HIV transmission to their HIV-negative partners. Recent experience with assisted reproductive technology from Europe and the United States reports $3390,{ }^{14} 2683^{15}$ and $420^{16}$ cycles of treatment with no seroconversions among women with 6-month follow-up HIV testing data. These figures suggest that the risk of HIV transmission occurring would be less than 1/6000 with assisted reproduction, which is lower than the estimated risk of sexual transmission per single act of penile-vaginal intercourse $(1 / 1000){ }^{17}$

A meta-analysis reported an overall HIV transmission rate of 0.46 per 100 person-years (irrespective of viral load), based on five episodes of HIV seroconversion. ${ }^{18}$ There were no data on viral load available for these five seroconversions, which occurred despite receipt of ART, and insufficient data to calculate rates according to condom use, sexually transmitted 
infections or for female-male and male-female transmission. ${ }^{18}$ When comparing overall transmission rates between patients who were and were not receiving ART, heterosexual transmission was reduced by $92 \%$ in those receiving ART. ${ }^{18}$ However, despite this reduction, there are case reports of HIV transmission occurring between serodiscordant individuals where the HIV-positive partner is receiving combination ART. ${ }^{19,20}$

Previous studies have reported abnormalities in semen parameters of HIV-positive men, including reduced volume, ${ }^{21-24}$ concentration ${ }^{21-24}$ and motility, $21,23,24$ and changes in morphology. ${ }^{23}$ There have been conflicting reports of the effect of combination ART on semen parameters, ${ }^{23,24}$ but many studies are small, and teasing out the effect of HIV and/ or the effect of antiretroviral drugs can be difficult. In our study, the only significant difference in sperm quality between HIV-positive clients receiving combination ART and the control group was in sperm velocity. Numbers in our study were too small to draw meaningful conclusions about any relationship between the specific combination ART regimen and semen abnormality. We also had no control group of HIV-positive men not receiving ART with whom to compare semen quality.

The relative contributions that HIV infection and ART make to semen quality remain unclear. Even if combination ART is found to be the major contributing factor to semen abnormality, the relative reduction in semen quality must be weighed against the potential benefit of reducing infectivity by reducing HIV viral load in semen. Along with the evidence of discordant HIV results between semen and plasma in men receiving combination ART, the observation of significantly impaired sperm velocity in our HIV-positive group supports a recommendation that serodiscordant couples attempting to conceive naturally should undergo semen analysis, and HIV-negative women should be educated regarding cycle tracking to maximise the chance of success.

Acknowledgements: In addition to the authors members of the Chronic Viral Illness (CVI) Study Group include Samantha Perna, Suzanne Garland, Suzanne Crowe and Janell Archer.

Competing interests: No relevant disclosures.

Received 15 Feb 2011, accepted 17 Aug 2011.

1 Joint United Nations Programme on HIV/AIDS (UNAIDS). 2008 Report on the global AIDS epidemic. Geneva: UNAIDS, 2008.

2 Chama C, Morrupa J, Gashau W. Sex and reproduction among HIV-infected people in Maiduguri, Nigeria. J Obstet Gynaecol 2007; 27: 812-815.

3 Chen JL, Philips KA, Kanouse DE, et al. Fertility desires and intentions of HIV-positive men and women. Fam Plann Perspect 2001; 33: 144-165.

4 Nobrega AA, Oliveira FA, Galvao MT, et al. Desire for a child among women living with HIV/AIDS in northeast Brazil.AIDS Patient Care STDS 2007; 21: 261-267.

5 Panozzo L, Battegay M, Friedl A, et al. High risk behaviour and fertility desires among heterosexual HIV-positive patients with a serodiscordant partner - two challenging issues. Swiss Med Wkly 2003; 133: 124-127.

6 Wesley Y. Desire for children among black women with and without HIV infection. J Nurs Scholarsh 2003; 35: 37-43.

7 Vernazza P, Hirschel B, Bernasconi E, Flepp M. [HIV infected people free of other STDs are sexually not infectious on effective antiretroviral therapy] [German]. Schweiz Arzteztg 2008; 89: 165-169.

8 Centers for Disease Control and Prevention (US). CDC underscores current recommendation for preventing HIV transmission [CDC statement]. Feb 2008. http://www.cdc.gov/hiv/resources/ press/020108.htm (accessed Oct 2011).

9 Wilson DP, Law MG, Grulich AE, et al. Relation between HIV viral load and infectiousness: a model-based analysis. Lancet 2008; 372: 314320.

10 Baker HWG, Mijch A, Garland S, et al. Use of assisted reproductive technology to reduce the risk of transmission of HIV in discordant couples wishing to have their own children where the male partner is seropositive with an undetectable viral load. J Med Ethics 2003; 29 : 315-320.

11 Bourne H, Archer J, Edgar DH, Baker HWG. Sperm preparation techniques. In: Gardner DK, Weissman A, Howles CM, Shoham Z, editors.
Textbook of assisted reproductive technologies: laboratory and clinical perspectives. 3rd ed. London: Informa Healthcare, 2008: 53-66.

12 Garrett C, Liu DY, Clarke GN, et al. Automated semen analysis: 'zona pellucida preferred' sperm morphometry and straight-line velocity are related to pregnancy rate in subfertile couples. Hum Reprod 2003; 18: 1643-1649.

13 Sullivan EA, Wang YA, Chambers G. Assisted reproductive technology in Australia and New Zealand 2008. Canberra: AlHW, 2010. (AlHW Cat. No. PER 49.)

14 Bujan L, Hollander L, Coudert M, et al. Safety and efficacy of sperm washing in HIV-1serodiscordant couples where the male is infected: results from the European CREAThE network. AIDS 2007; 21: 1909-1914.

15 Savasi V, Ferrazzi E, Lanzani C, et al. Safety of sperm washing and ART outcome in $741 \mathrm{HIV}-1$ serodiscordant couples. Hum Reprod 2007; 22 : 772-777.

16 Sauer MV, Wang JG, Douglas NC, et al. Providing fertility care to men seropositive for human immunodeficiency virus: reviewing 10 years of experience and 420 consecutive cycles of in vitro fertilization and intracytoplasmic sperm injection. Fertil Steril 2009; 91: 2455-2460.

17 de Vincenzi I; European Study Group on Heterosexual Transmission of HIV. A longitudinal study of human immunodeficiency virus transmission by heterosexual partners. N Engl J Med 1994; 331: 341-346.

18 Attia S, Egger M, Muller M, et al. Sexual transmission of HIV according to viral load and antiretroviral therapy: systematic review and meta-analysis. AIDS 2009; 23: 1397-1404.

19 Sullivan P, Kayitenkore K, Chomba E, et al. Is the reduction of HIV transmission risk while prescribed antiretroviral therapy (ARVT) different for men and women? Results from discordant couples in Rwanda and Zambia. Abstract no. WEAC101. 5th IAS Conference on HIV Pathogenesis, Treatment and Prevention; 2009 Jul 19-22; Cape Town, South Africa.

20 Sturmer M, Doerr HW, Berger A, Gute P. Is transmission of HIV-1 in non-viraemic serodiscordant couples possible? Antivir Ther 2008; 13: 729-732

21 Muller CH, Coombs RW, Krieger JN. Effects of clinical stage and immunological status on semen analysis results in human immunodeficiency virus type 1-seropositive men. Andrologia 1998; 30 Suppl 1: 15-22.

22 Dulioust E, Du AL, Costagliola D, et al. Semen alterations in HIV-1 infected men. Hum Reprod 2002; 17: 2112-2118.

23 Nicopoullos JDM, Almeida P, Vourliotis M, GillingSmith C. A decade of the sperm-washing programme: correlation between markers of HIV and seminal parameters. HIV Med 2011; 12: 195201.

24 Bujan L, Sergerie M, Moinard N, et al. Decreased semen volume and spermatozoa motility in HIV-infected patients under antiretroviral treatment. J Androl 2007; 28: 444-452. 